\title{
A Novel Rehabilitative Protocol in the Treatment of Mixed Urinary Incontinence in Women: The Effects of Focused Mechano-Acoustic Vibration
}

\author{
Teresa Paolucci, ${ }^{1, *}$ Rosa Grazia Bellomo, ${ }^{2}$ Letizia Pezzi, ${ }^{1}$ Franco Frondaroli, ${ }^{3}$ Serena Frondaroli, ${ }^{4}$ Alessandro Santarelli, ${ }^{3}$
} Claudia Barbato, ${ }^{2}$ Annamaria Porreca, ${ }^{5}$ and Raoul Saggini ${ }^{1}$

\begin{abstract}
Dysfunctions of the pelvic floor related to mixed urinary incontinence in women are pathologies extremely limiting for patients bodily and psychosocial conditions, altering their quality of life. The aim of this study was to determine the effects of focal mechanical vibrations in mixed urinary incontinence. In this retrospective observational case-control study, 65 patients were randomized and divided into 2 groups: treatment group by focal mechanical vibrations (VISS-10 sessions) $(N=33)$ and a control group in waiting list $(N=32)$. Also, both groups received home-based postural ergonomic instructions to reinforce pelvic floor. Data were collected at T0 (baseline), $\mathrm{T} 1$ (end of treatment), and T2 (follow-up = after 1 month): rheological muscle parameters were assessed by MyotonPRO respect to evaluate the gluteus maximus muscle. Then, to measure the general disability of the pelvic floor and the impact of urogenital problems on daily activities the Pelvic Floor Disability Index (PFDI-20) and the Pelvic Floor Impact Questionnaire (PFIQ-7) were used. Groups were matched perfectly before treatment for age (58.20 \pm 4.37 vs. $58.73 \pm 5.19)$ and BMI $(26.15 \pm 2.22$ vs. $25.85 \pm 2.11)$; for the two-way ANOVA analysis, a difference in gluteus variables over time and between groups except for GMDR (group $p$-value $=0.60$ ) was showed The two-way ANOVA shows statistically significant effects of treatment and time for PDFI-20 and PFIQ-7 ( $p$-value $<0.001$ ). An improvement in incontinence symptoms and quality of life in the PDFI-20 and PFIQ-7 scores were reported and VISS may favor muscles stiffness for exercises by improving the normalization of basal tone. Our results were encouraging and suggested the use of focal mechanical vibration as a novel tool for treating mix urinary incontinence in women to complete and help the rehabilitative therapeutic protocol.
\end{abstract}

Keywords: exercise; pelvic muscle training; physical therapy; posture; rehabilitation; urinary incontinence

\section{Introduction}

Urinary female incontinence, defined by the International Continence Society as the complaint of any involuntary leakage of urine, is a pathology that has significant implications for patients and their families and a tremendous economic impact on health ser- vices. ${ }^{1,2}$ There are various forms of urinary incontinence, each of which is identified specifically with regard to symptoms and causes: stress urinary incontinence (SUI, involuntary leakage on effort or exertion or on sneezing or coughing), urge incontinence (UI, an involuntary leakage that is accompanied or immediately

\footnotetext{
${ }^{1}$ Unit of Physical Medicine and Rehabilitation, Department of Medical and Oral Sciences and Biotechnologies (DSMOB), G. d'Annunzio University of Chieti-Pescara, Chieti, Italy.

${ }^{2}$ Department of Biomolecular Science, Physical Medicine and Rehabilitation, Carlo Bo University Study of Urbino, Urbino, Italy.

${ }^{3}$ Obstetric-Gynecological Clinic (Maternal-Infant Department), University Hospital of "SS. Annunziata", Chieti, Italy.

${ }^{4}$ Complex Gynecological Operative Unit, "Floraspe Renzetti" Hospital, Lanciano, Italy.

${ }^{5}$ Department of Economic Science, G. d'Annunzio University, Chieti-Pescara, Italy.

*Address correspondence to: Teresa Paolucci, MD, PhD, Unit of Physical Medicine and Rehabilitation, Department of Medical and Oral Sciences and Biotechnologies (DSMOB), G. d'Annunzio University of Chieti-Pescara, Via dei Vestini 21, Chieti 66100, Italy, E-mail: teresapaolucci@hotmail.com
}

(C) Teresa Paolucci et al. 2019; Published by Mary Ann Liebert, Inc. This Open Access article is distributed under the terms of the Creative Commons License (http://creativecommons.org/licenses/by/4.0), which permits unrestricted use, distribution, and reproduction in any medium, provided the original work is properly cited. 
preceded by urgency), mixed incontinence (MI, involuntary leakage that is associated with urgency, exertion, effort, sneezing, or coughing), and incontinence due to regurgitation. ${ }^{3-5}$

However, urinary incontinence is a debilitating problem, because it prevents daily activities and disrupts the psychological well-being of women who are affected, especially those aged 65 years or older. ${ }^{6}$ It is often caused by a weakening of the pelvic floor, blockage of the urethra, or a decrease in the sphincter tone of the urethra, but there are other causes, such as urinary or vaginal infections, secondary effects of certain drugs, muscle weakness, nerve or muscle diseases, and secondary effects of surgical procedures. ${ }^{7-9}$

Urinary incontinence can be treated successfully, often by combining several approaches, such as surgical therapy, pharmacological therapy, and pelvic reeducation. ${ }^{10,11}$

Moreover, compared with stress incontinence (SI), women with UI, and MI report significantly higher ratings of urinary urge intensity, more incontinence episodes, and worse in quality of life. ${ }^{12}$ The higher-thanexpected prevalence of MI could be due to SI and UI episodes occurring at separate times but being linked by a common predisposing factor. ${ }^{13,14}$ Clinical practice guidelines recommend individualized pelvic floor muscle (PFM) training as a first-line treatment for stress or mixed UI in women, although the lack of personnel and financial resources has limited the delivery of this modality. ${ }^{11}$

Alternatively, or in parallel with any type of surgical or pharmacological intervention, a physical rehabilitation approach, known as pelvic re-education, is based on the possibility of urging and accustoming the patient to self-management of muscular contractions. Muscles, such as the adductor magnus (AM), gluteus maximus (GM), rectus abdominis (RA), and abdominal external and internal oblique muscles, have important functions in the treatment of SUI, and a relationship between the contraction of these muscles and PFMs has been established. ${ }^{15,16}$ In addition, the co-activation of abdominal and PFMs is significant in regulating internal abdominal pressure, which is critical for proper continent function.

The nature of this re-education is above all preventive, but the progress in technology has meant that in certain cases, pelvic rehabilitation can be implemented as auxiliary healing therapy. In rehabilitation, among physical therapies using medical devices, mechanical focal vibration has generated good results with regard to muscle strengthening. ${ }^{17}$ Also, using high-frequency focal vibration, Saggini et al. normalized the basal muscle tone in healthy persons. ${ }^{18}$ No study has examined the use of focal mechanical vibration in the reinforcement and normalization of tone and stiffness in the floor and pelvic girdle muscles that are implicated in mixed urinary incontinence (MUI) incontinence in women.

Based on this premise, the aim of this study was to determine the effects of focal mechanical vibrations in MUI. The primary outcome was the improvement in symptoms per the Pelvic Floor Disability Index (PFDI-20), ${ }^{19}$ and the secondary outcome was the evaluation of tone and stiffness of the GM muscle.

\section{Materials and Methods}

This retrospective observational case-control study examined the effects of focused mechano-acoustic vibration therapy (VISS) in the rehabilitation of MUI in women per the Strobe Guidelines. ${ }^{20}$

Seventy-six $(N=76)$ patients with MUI were recruited from the gynecology outpatient clinic of G. D'Annunzio University of Chieti-Pescara; 74 patients agreed to participate, and 65 met the inclusion criteria and were enrolled, visiting the physical medicine and rehabilitation outpatient clinic of this institution from June 2018 to January 2019.

Sample randomization (1:1) was performed, and the patients were divided into two groups: treatment with VISS (EX) $(N=33)$ and a wait list $(\mathrm{CT})(N=32)$.

The inclusion criteria were age between 50 and 70 years; a diagnosis of MUI, as defined by the International Continence Society (ICS) ${ }^{4}$; and body mass index $(\mathrm{BMI})<30$. The exclusion criteria were participation in other therapeutic protocols and the presence of urogenital infections, neurological pathologies, pelvic organ prolapse, pain, hematuria, voiding symptoms, and spinal lesions of any grade. Women with prior anti-incontinence surgery or medical treatment for overactive bladder were excluded. The clinician (a specialist in gynecology) assessed the predominant type of incontinence and its duration and performed a urine analysis and urine culture. The physical examination included abdominal, pelvic, and vaginal examinations and a stress test.

This study was performed per the Helsinki Declaration on human experimentation and was approved by the Departmental committee of G. D'Annunzio University of Chieti (Italy). All patients gave written informed consent after receiving detailed information on the study's aims and procedures. Patients were 
evaluated at T0 (before the start the protocol), T1 (after completion of the rehabilitative protocol), and T2 (1-month follow-up).

In addition, patients were asked to report and record any adverse effects during the rehabilitative treatment, daytime and night-time losses, and intervals that were free from urine leakage (hours), quantifying urine loss (drops per day) in an individual voiding diary.

\section{Outcome measures}

To measure rheological muscle parameters, a MyotonPRO ${ }^{21-23}$ was used to evaluate the GM. ${ }^{15}$ Specifically, the following parameters were considered: muscular tone (F-oscillation frequency), to indicate the basal involuntary mechanic tension of the muscle, expressed in Hertz $(\mathrm{Hz})$; muscular elasticity/plasticity (D-logarithmic decrement), as the capacity of a muscle to recover its initial shape after mechanical modification; and muscular resistance (S-dynamic stiffness), as the property of the muscle to counteract external mechanical deformation through the activation of antagonist muscles (this value correlates with muscular tone and is expressed in $\mathrm{N} / \mathrm{m}$ ).

These measurements were made by applying the needle-shaped pressure sensor of the instrument to the center of the selected muscle belly. The instrument, after repeating 3 percussions on the muscle, yielded values regarding its tone, elasticity, and stiffness, also calculating the probability of error of the measurement. The accepted probability of error was set to $2 \%$.

The PFDI-20 measures the general disability level of the pelvic floor, considering symptoms over the past 3 months before the evaluation. It is composed of three subscales:POPDI-6, CRADI-8, and UDI-6. The score for each item varies from 0 (no symptoms) to 4 points (extremely disabling symptoms). For each scale, the average score of the answers is calculated (ranging from 0 to 4 points) and multiplied by $25(100 / 4)$ to obtain the final score of the subscale (from 0 to 100). The final PFDI-20 score is derived from the sum of each subscale (between 0 and 300 points). ${ }^{19}$

The Pelvic Floor Impact Questionnaire (PFIQ-7) determines the impact of urogenital problems on daily activities, considering symptoms from the 3 months before evaluation. It comprises seven items, each of which is related to a specific urogenital symptom. The score for each item varies from 0 (absent) to 3 points (extremely present). Each symptom is assessed regarding its presence at the level of the bladder/ urine (UIQ-7), intestine/rectus (CRAIQ-7), and vagi- na/pelvis (POPIQ-7). For each subscale, the mean score is calculated (from 0 to 3 points) and multiplied by $33.3(100 / 3)$ to obtain the final score for the subscale (0-100 points). The final PFIQ-7 score is derived from the sum of each subscale (0-300 points). ${ }^{24}$

\section{Rehabilitative treatment group}

The experimental therapeutic protocol (EX), consisted of 10 sessions, 3 times per week for the first 2 weeks and then twice weekly for the next 2 weeks. A frequency of $300 \mathrm{~Hz}$ for $15 \mathrm{~min}$ was used for every muscle that was treated by the expert physiotherapist during the treatment. Finally, a follow-up period of 1 month ensued without treatment. Each patient underwent focused mechanoacoustic vibration with applicators placed bilaterally at the level of the rectus abdominis, adductor muscles (gracilis, pectineus, long adductor, and short adductor muscles), GM, quadratus lumborum, and perineal area (Fig. 1).

The focused mechano-acoustic vibration was administered using the Vibration Sound System ${ }^{\circledR}$ (European patent: Ep1824439-CE 1936 Certificate of Conformity - $N^{\circ}$ HD 60114019 - Unibell, Calco - LC, Italy) (Fig. 2). This instrument consists of a 32,000revolution turbine with a flow rate of $35 \mathrm{~m}^{3} / \mathrm{h}$ that generate air waves with a pressure of up to 250 mbar and a flow modulator that vibrates air with a pressure of up to $630 \mathrm{mbar}$ and frequency of up to $980 \mathrm{~Hz}$ (however, a frequency up to $300 \mathrm{~Hz}$ is recommended) for producing mechano-acoustic waves. ${ }^{25}$

Whole patients as in the EX and CT (waiting list) received postural ergonomic instructions that were to be put into practice in everyday life to improve urinary incontinence symptoms; trained with respect to isometric contraction exercises for the GM, tilting exercises for the pelvis, and diaphragmatic breathing exercises; and instructed to walk at least 120 min per week. Every patient was given a pamphlet to explain the home exercises (Fig. 3).

\section{Statistical evaluation}

Sample size calculation. The following conditions were set to determine the sample size: a significance level of $95 \%$ and a power of $80 \%$ under the hypothesis of an average variation in PFDI-20 scores $^{19}$ of $60.00 \pm$ 19.19 for EX (VISS) versus CT (wait list) after treatment. As a result, 30 patients for each group were enrolled, considering a dropout rate of $20 \%$.

Data analysis. Data were collected at T0, T1, and T2 to determine how the patients, regardless of treatment, 


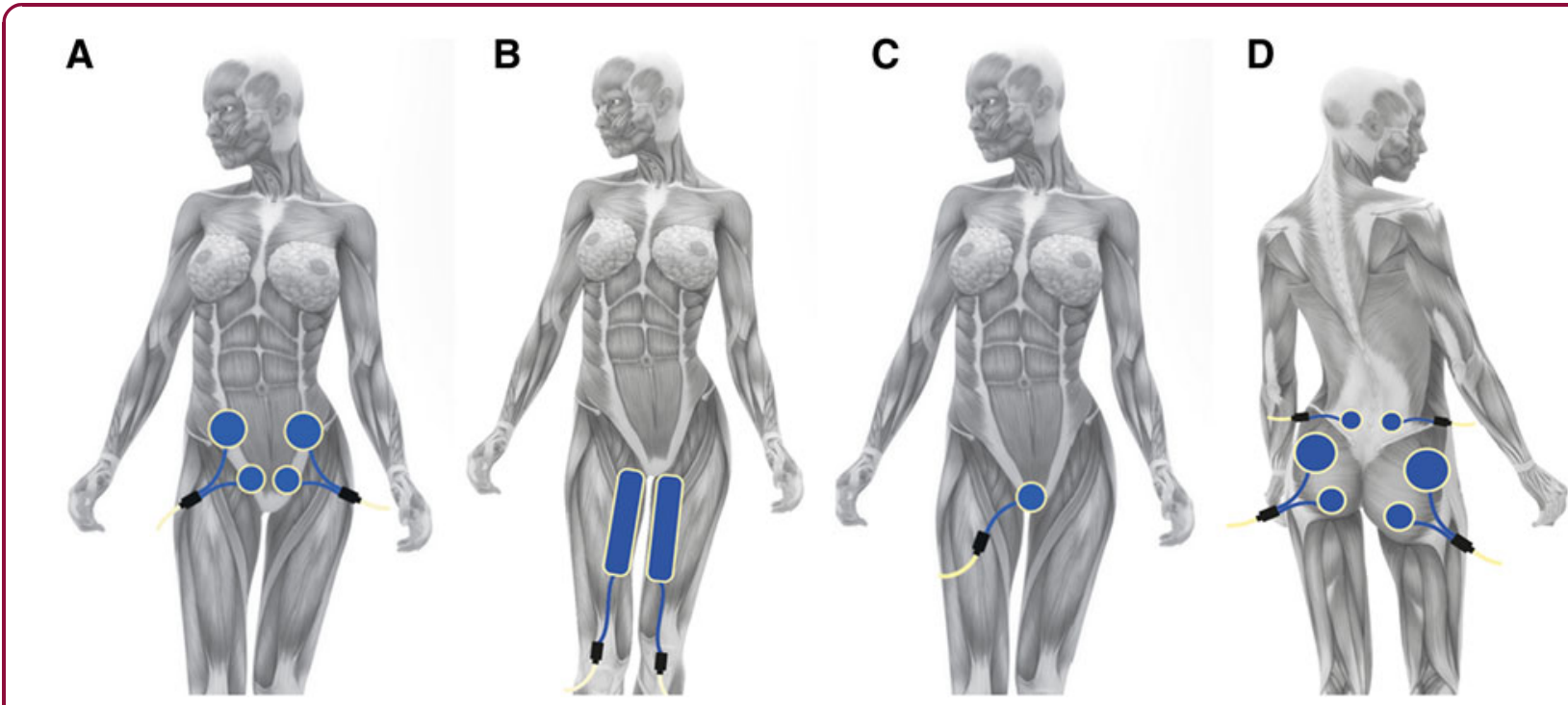

FIG. 1. (A-D) The anatomic model shows the positioning of the transducers by VISS with respect to the aforementioned reference muscles. (A) abdominal muscles; (B) adductors muscles; (C) pelvic floor muscle; (D) gluteus maximus muscle and quadratus lumborum muscles.

improved their conditions. Differences in weight, age, BMI, and height were analyzed by $t$-test. The normality of the data distribution was examined by Jarque-Bera test, and covariance sphericity was analyzed by Mauchly test- Differences in the mean values for the gluteus maximum (right and left sides- $\mathrm{R}$ and $\mathrm{L}$ ) variables (muscular tone-F, muscular elasticity/plasticity$\mathrm{D}$, and muscular resistance-S) in each group over time were assessed by two-way ANOVA for repeated measurements, followed by Tukey's post hoc test:
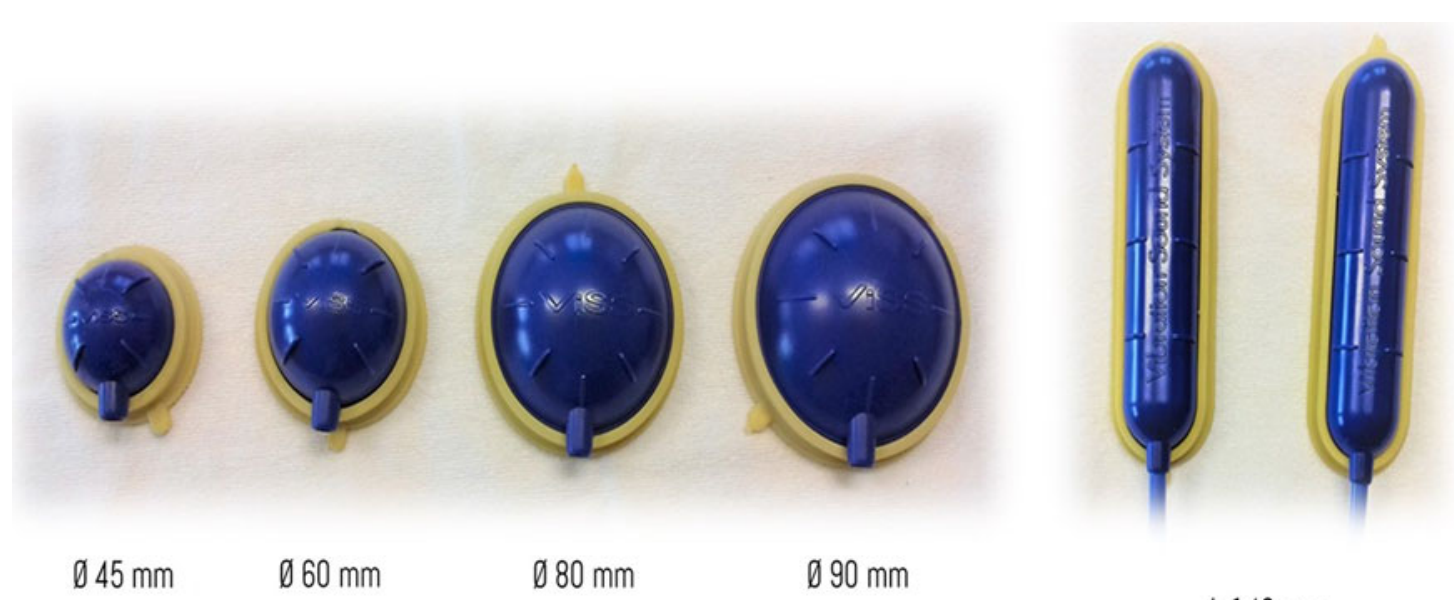

$\mathrm{L} 140 \mathrm{~mm}$

FIG. 2. VISS transducers. The transducers are of different shape and size; they are composed of ABS, a common thermoplastic polymer with mechanical properties as impact and heat resistance and toughness, while the lateral bearing is made of Santoprene TPV. ABS, acrylonitrile butadiene styrene. 
1

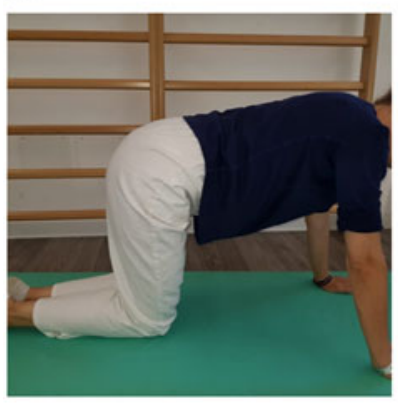

4

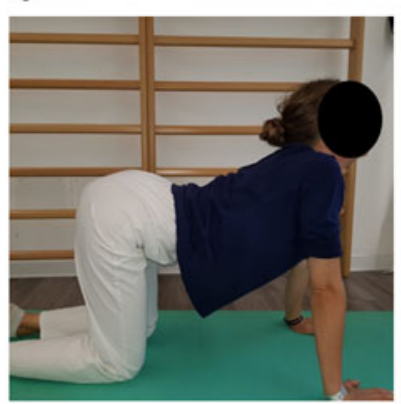

7

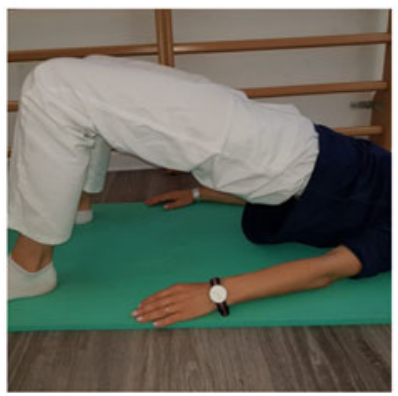

2

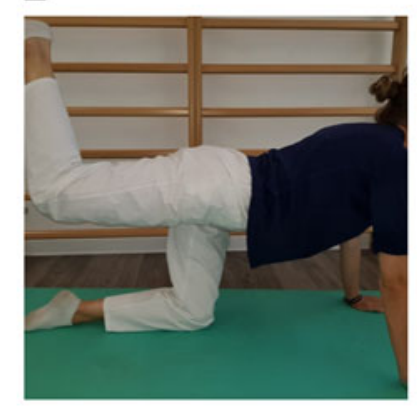

5

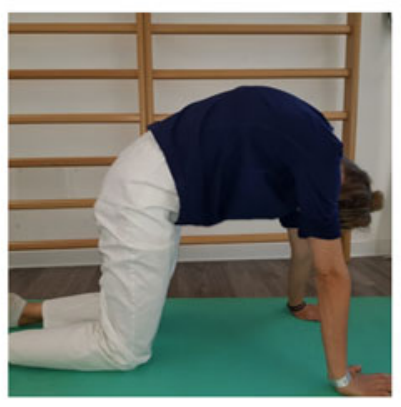

8

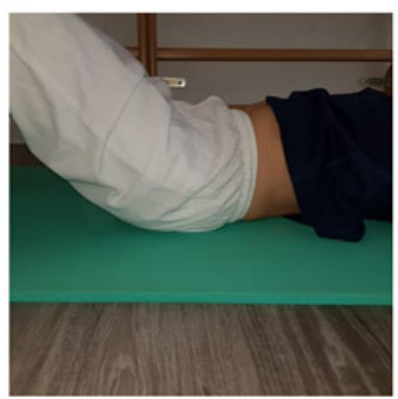

3

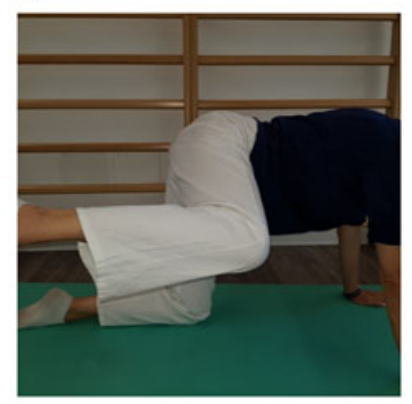

6

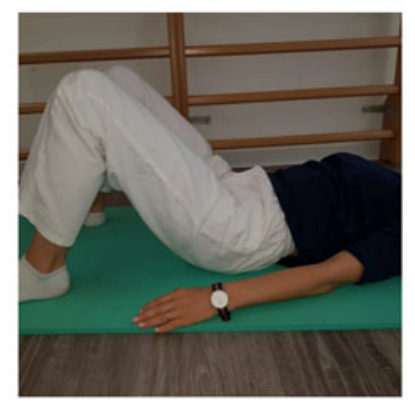

9

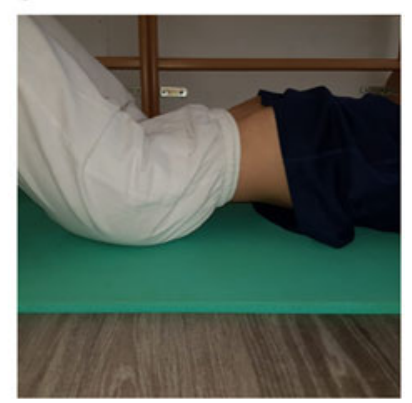

FIG. 3. Home-made exercises. Exercises 1-2-3=exercises to strengthen gluteus muscles (hip kicker); Exercises $4-5=$ exercises to strengthen upper and lower back muscles (angry cat stretch); Exercises $6=$ abdominal breathing; Exercises 6-7=exercises to strengthen gluteus muscles and pelvic floor (basic bridge); Exercises $8-9=$ exercises to strengthen pelvic floor (pelvic tilt).

$\mathrm{GMD}=$ gluteus maximus muscle logarithmic decrement; GMF = gluteus maximus muscle oscillation frequency $[\mathrm{Hz}]$; GMS = gluteus maximus muscle dynamic stiffness $[\mathrm{N} / \mathrm{m}]$.

Except for oscillation frequency right (GMFR) for the gluteus maximus, the variables violated the normality and sphericity assumptions; thus, these data were subjected to Tukey's ladder of powers transformation. Further, the PFDI-20 and PFIQ-7 scales were tested by repeated measures two-way ANOVA.
Before parametric analysis was performed, descriptive statistics were used to describe the sample. Differences were evaluated at $p$-value $<0.05$. All analyses were performed in $\mathrm{R}$ environment, and the sample size was calculated with G-power, version 3.1.

\section{Results}

The data of the 60 patients were calculated (Fig. 4). Tables 1 and 2 lists the descriptive statistics for each variable (clinical scales and MyotonPro values) in the 


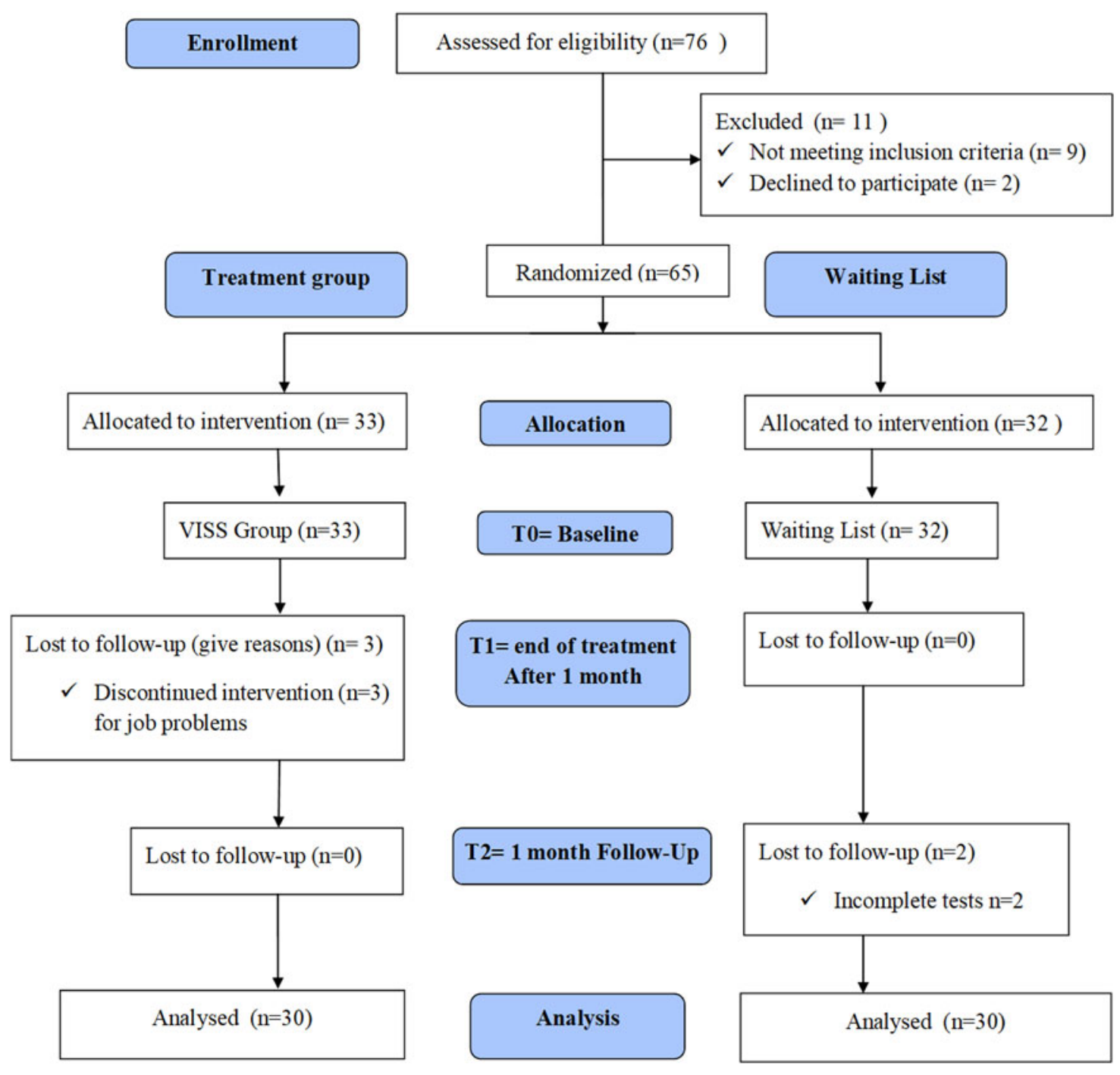

FIG. 4. Flowchart.

two groups. Groups were matched perfectly before treatment for age and BMI, and the means for the groups did not differ statistically at baseline: the mean age of the EX was $58.20 \pm 4.37$ years versus $58.73 \pm 5.19$ years in the CT; for mean BMI $\left(\mathrm{kg} / \mathrm{m}^{2}\right)$

Table 1. Mean and SD for Every Variable in the Control (CT) and Treatment (EX) Groups

\begin{tabular}{lrrr}
\hline \multicolumn{2}{c}{ Control group (CT) } & & Treatment group (EX) \\
Variable & Mean \pm SD & & Mean \pm SD \\
\hline Age (years) & $58.20 \pm 4.37$ & & $58.73 \pm 5.19$ \\
Weight $(\mathrm{kg})$ & $72.23 \pm 9.03$ & & $71.47 \pm 9.15$ \\
Hight $(\mathrm{m})$ & $1.66 \pm 0.06$ & & $1.66 \pm 0.06$ \\
$\mathrm{BMI}\left(\mathrm{kg} / \mathrm{m}^{2}\right)$ & $26.15 \pm 2.22$ & & $25.85 \pm 2.11$ \\
\hline
\end{tabular}

in the EX and CT was $26.15 \pm 2.22$ and $25.85 \pm 2.11$, respectively.

Table 3 shows the results of the two-way ANOVA, confirming the differences in gluteus variables over time and between groups except for GMDR (group $p$-value $=0.60)$. The principal effects were statistically significant, but their interaction was not. Thus, for every variable, except GMDR, being treated or not had a different effect on the outcome over time. Table 4 shows the $p$-values and effect sizes for PDFI20 and PFIQ-7, which were statistically significant with regard to the principal effects and their interaction, indicating that there were significant differences between groups and over time. Thus, the change in 
Table 2. Mean and SD for Outcome Measures in the Control (CT) and Treatment (EX) Groups

\begin{tabular}{|c|c|c|c|c|c|c|}
\hline \multirow[b]{2}{*}{ Variable } & \multicolumn{2}{|c|}{ T0 } & \multicolumn{2}{|c|}{$\mathrm{T} 1$} & \multicolumn{2}{|c|}{$\mathrm{T} 2$} \\
\hline & EX & CT & EX & CT & EX & $\mathrm{CT}$ \\
\hline GMDL & $1.60 \pm 0.28$ & $1.55 \pm 0.28$ & $1.74 \pm 0.28$ & $1.68 \pm 0.34$ & $1.77 \pm 0.28$ & $1.69 \pm 0.35$ \\
\hline GMDR & $1.57 \pm 0.27$ & $1.60 \pm 0.29$ & $1.74 \pm 0.29$ & $1.71 \pm 0.34$ & $1.77 \pm 0.30$ & $1.72 \pm 0.34$ \\
\hline GMFL (Hz) & $13.02 \pm 1.13$ & $13.15 \pm 1.46$ & $11.77 \pm 1.53$ & $12.39 \pm 1.69$ & $11.76 \pm 1.57$ & $12.49 \pm 1.75$ \\
\hline GMFR (Hz) & $13.02 \pm 1.04$ & $13.17 \pm 1.2$ & $11.68 \pm 1.30$ & $12.25 \pm 1.72$ & $11.73 \pm 1.40$ & $12.32 \pm 1.74$ \\
\hline GMSL (N/m) & $251.11 \pm 24.56$ & $307.81 \pm 20.53$ & $226.77 \pm 20.24$ & $300.23 \pm 19.99$ & $230.01 \pm 19.2$ & $300.40 \pm 19.67$ \\
\hline GMSR (N/m) & $222.39 \pm 18.10$ & $226.59 \pm 22.11$ & $212.5 \pm 16.19$ & $220.83 \pm 22.08$ & $213.16 \pm 15.53$ & $221.62 \pm 21.48$ \\
\hline PFDI-20 (0-300) & $77.13 \pm 21.17$ & $77.30 \pm 23.74$ & $49.20 \pm 17.18$ & $79.20 \pm 21.11$ & $53.83 \pm 19.22$ & $77.43 \pm 21.74$ \\
\hline PFIQ-7 (0-300) & $110.93 \pm 24.88$ & $109.60 \pm 27.13$ & $68.17 \pm 24.14$ & $110.93 \pm 24.88$ & $71.27 \pm 21.74$ & $117.63 \pm 30.17$ \\
\hline
\end{tabular}

GMDL, gluteus maximus muscle logarithmic decrement Left; GMDR, gluteus maximus muscle logarithmic decrement Right; GMFL, gluteus maximus muscle oscillation frequency Left; GMFR, gluteus maximus muscle oscillation frequency Right; GMSL, gluteus maximus muscle dynamic stiffness Left; GMSR, gluteus maximus muscle dynamic stiffness Right; PFDI-20, Pelvic Floor Disability Index; PFIQ-7, Pelvic Floor Impact Questionnaire.

variables over time differed, depending on group membership. Figure 5 shows the trends of PDFI-20 and PFIQ-7.

No adverse effects were reported during the rehabilitative treatment.

\section{Discussion}

The main finding of this study was the positive effect of VISS on reducing disability index scores with respect to MUI in the EX versus CT. In particular, in the VISS group, patients reported improvements in their urinary incontinence symptoms and quality of life, their PDFI-20 and PFIQ-7 scores were statistically significant, and the change over the three evaluation times differed, depending on group membership.

The patients' perception of their disability with regard to MUI became positive, the EX group received intensive treatment, at least in the first 2 weeks, likely ensuring greater compliance with the rehabilitation with VISS continuity with the home-based exercises. The association between VISS and exercises that reinforce the GM muscle, tilting exercises for the pelvis, diaphragmatic breathing exercises, and instructions to walk at least $120 \mathrm{~min}$ per week showed encouraging positive results in improving MUI. Compared with previous studies, ${ }^{25,26}$ mechanical focal vibration can be a good approach for rehabilitating the PFMs; it is also simpler and less invasive than other techniques used.

VISS is a new approach to MUI, the mechanism of action on the PFMs and other muscles that could be attributed to the stimulation of mechanoreceptors, in particular the Pacinian corpuscles, which have the highest sensitivity $(1 \mathrm{~mm})$ at a frequency of $250-300 \mathrm{~Hz}$, thus representing the target vibration receptors, ${ }^{27}$ and the consequent muscle training due to a frequency of $300 \mathrm{~Hz}$, as in our rehabilitation protocol. ${ }^{28,29}$

Also, other groups have reported that high-frequency focal vibration induces long-term reorganization of the primary motor cortex, ${ }^{17,30-32}$ characterized by persistent increases in intracortical and cortical reciprocal inhibition, which might reduce undesired muscle activation and minimize co-contractions, consequently enhancing motor performance. We hypothesize that this mechanism would allow better integration between the PFMs and pelvis-stabilizing muscles, such as the rectus abdominis, in controlling the posterior tilting of the pelvis, GM, and large adductor, such as synergistic muscles of the rectus abdominis. During the Valsalva maneuver, incontinent women experience greater anteroposterior displacement than continent women, and

Table 3. Two-Way ANOVA of Gluteus Muscle Variables

\begin{tabular}{|c|c|c|c|c|c|c|}
\hline Factors & GMDL & GMDR & GMFL & GMFR & GMSL & GMSR \\
\hline \multicolumn{7}{|c|}{$p$-values (Effect size) } \\
\hline Group & $<0.05(0.023)$ & $0.60(0.002)$ & $<0.05(0.023)$ & $<0.05(0.021)$ & $<0.001(0.698)$ & $<0.05(0.029)$ \\
\hline Time & $<0.01(0.057)$ & $<0.01(0.055)$ & $<0.001(0.095)$ & $<0.001(0.118)$ & $<0.001(0.030)$ & $<0.05(0.044)$ \\
\hline Interact. & $0.89(0.001)$ & $0.71(0.004)$ & $0.44(0.008)$ & $0.63(0.004)$ & $0.19(0.005)$ & $0.63(0.005)$ \\
\hline
\end{tabular}

GMDL, gluteus maximus muscle logarithmic decrement Left; GMDR, gluteus maximus muscle logarithmic decrement Right; GMFL, gluteus maximus muscle oscillation frequency Left; GMFR, gluteus maximus muscle oscillation frequency Right; GMSL, gluteus maximus muscle dynamic stiffness Left; GMSR, gluteus maximus muscle dynamic stiffness Right. 
Table 4. Two-Way ANOVA of PDFI-20 and PFIQ-7

\begin{tabular}{lcc}
\hline Factors & PFDI-20 & PFIQ-7 \\
\hline$p$-values (Effect size) & & \\
Group & $<0.001(0.0142)$ & $<0.001(0.041)$ \\
Time & $<0.001(0.062)$ & $<0.001(0.063)$ \\
Interact. & $<0.001(0.078)$ & $<0.001(0.079)$ \\
\hline
\end{tabular}

PFDI-20, pelvic floor disability index; PFIQ-7, pelvic floor impact questionnaire.

the deep layer of the PFM is more tightly modulated by respiration than the superficial layer, but activation of the superficial layer was extensive during maximal/ submaximal occluded respiratory efforts and earlier during cough. ${ }^{33,34}$

Thus, in our rehabilitative protocol, importance was given to teaching and adopting diaphragmatic breathing in home-based exercises. Also, such muscles as the AM, GM, RA, and abdominal external and internal oblique muscles, are critical in the treatment of urinary incontinence: synergistic activation of these muscles intensifies a woman's ability to contract the PFM. $^{15}$

The objectives of our rehabilitative intervention were to promote awareness of the contraction of the pelvic muscles in patients; control and, if possible, eliminate the synergies between agonist and antagonist muscles that are involved in the biomechanics of the pelvic floor; and integrate the rehabilitative program with activities of daily living by promoting walking for at least 120 min per week.

Our results showed normalization of basal tone (F), plasticity (D), and stiffness (S) in the EX and CT with respect to the GM, which was used as the reference in the MyotonPro analysis. ${ }^{35}$ Thus, it is not possible to conclude that VISS is better than the home-based exercises that were proposed to the patients. Nevertheless, the EX experienced major changes in rheological properties of the muscle with versus the CT, except for GMDR (group $p=0.60$ ). We assume that VISS prepares muscles for home-based exercises by improving the normalization of basal tone.

The lack of a time-group interaction could be due to various factors: it was difficult to find homogeneity of the rheological parameters of the muscles at T0, which rendered the two groups perfectly comparable, and moreover, the MytonPro is useful in clinical studies, but it likely has lower sensitivity than surface electromyography, due to a bias that is linked to the repeatability of the measurements. ${ }^{15}$ Compared with

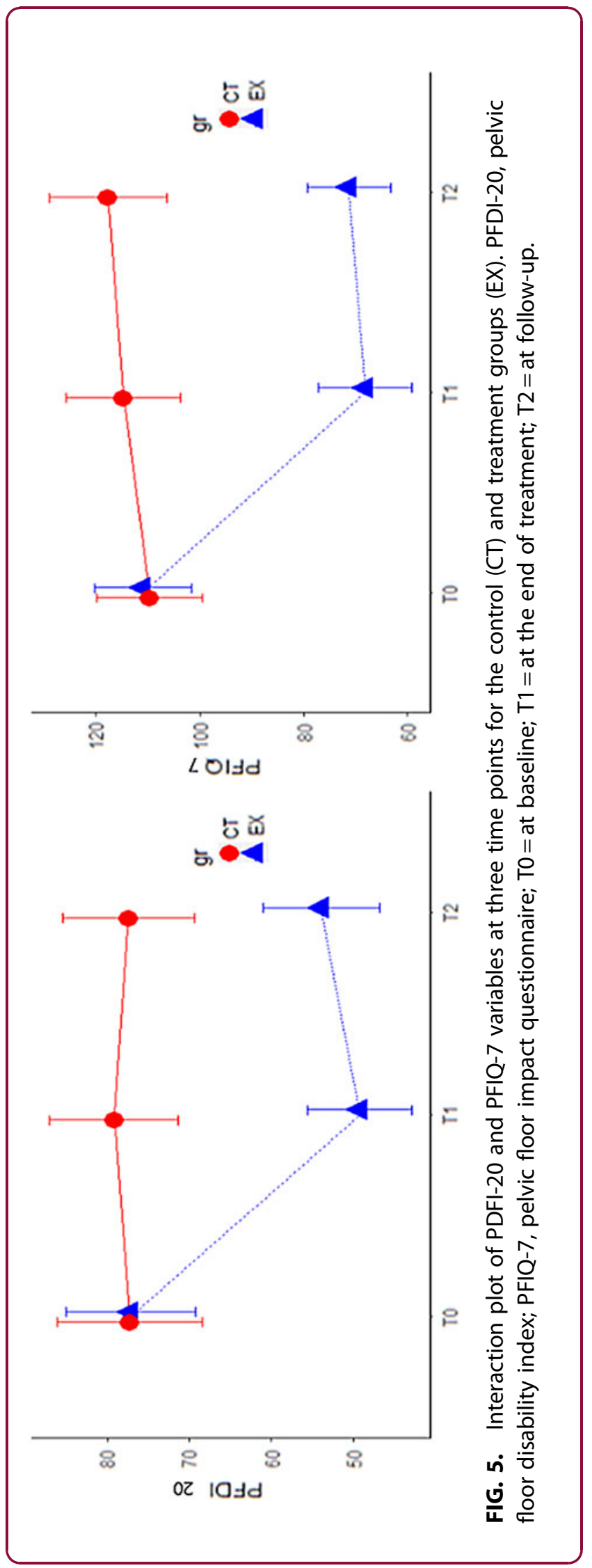


the clinical scales, patients with MUI episodes in the EX experienced significantly greater improvement versus the CT; the combination of VISS and home-based exercises could have effected better functional motor coordination of the PFMs and agonist muscles that were treated with focal mechanical vibration. A strength of this study is that it is the first attempt to use VISS for MUI. One weakness was the lack of a control group of healthy female participants for evaluating and comparing the rheological properties of the GM by MyotonPro analysis.

\section{Conclusion}

In conclusion, our results are encouraging and suggest the use of VISS by high-frequency focal vibration as a valid therapeutic tool for treating MUI in women. The prescription of simple home-based exercises for pelvic tilting and diaphragmatic breathing, which are involved in the control of urinary function and intraabdominal pressure, will allow the patient to become aware of the contraction of the PFMs.

\section{Author Disclosure Statement}

No competing financial interest to declare.

\section{Funding Information}

No funding was received for this article.

\section{References}

1. Abrams P, Cardozo L, Fall M, et al. The standardization of terminology of lower urinary tract function: report from the standardization sub-committee of the International Continence Society. Neurourol Urodyn. 2002; 21:167-178.

2. Payne CK. Epidemiology, pathophysiology, and evaluation of urinary incontinence and overactive bladder. Urology. 1998;51:3-10.

3. Wood LN, Anger JT. Urinary incontinence in women. BMJ. 2014;349: g4531.

4. Bandukwala NQ, Gousse AE. Mixed urinary incontinence: what first? Curr Urol Rep. 2015;16:9.

5. Committee on Practice Bulletins-Gynecology and the American Urogynecologic Society. ACOG Practice Bulletin No. 155: urinary Incontinence in Women. Obstet Gynecol. 2015;126:e66-e81.

6. Kwak Y, Kwon H, Kim Y. Health-related quality of life and mental health in older women with urinary incontinence. Aging Ment Health. 2016;20: 719-726.

7. Irwin GM. Urinary incontinence. Prim Care. 2019;46:233-242.

8. Özdemir K, Şahin S, Özerdoğan N, et al. Evaluation of urinary incontinence and quality of life in married women aged between 20 and 49 years (Sakarya, Turkey) Turk J Med Sci. 2018;48:100-109.

9. Hagan KA, Erekson E, Austin A, et al. A prospective study of the natural history of urinary incontinence in women. Am J Obstet Gynecol. 2018; 218:502.e1-e502.e8.

10. Betschart C, Mol SE, Lütolf-Keller B, et al. Pelvic floor muscle training for urinary incontinence: a comparison of outcomes in premenopausal versus postmenopausal women. Female Pelvic Med Reconstr Surg. 2013;19: 219-224.

11. Qaseem A, Dallas P, Forciea MA, et al. Clinical Guidelines Committee of the American College of Physicians. Nonsurgical management of urinary incontinence in women: a clinical practice guideline from the American College of Physicians. Ann Intern Med. 2014;161:429-440.
12. Coyne KS, Zhou Z, Thompson C, et al. The impact on health-related quality of life of stress, urge and mixed urinary incontinence. BJU Int. 2003;92:731-735.

13. Dallosso HM, McGrother CW, Matthews RJ, et al. The association of diet and other lifestyle factors with overactive bladder and stress incontinence: a longitudinal study in women. BJU Int. 2003;92:69-77.

14. Brown JS, Grady D, Ouslander JG, et al. Prevalence of urinary incontinence and associated risk factors in postmenopausal women. Heart \& Estrogen/ Progestin Replacement Study (HERS) Research Group. Obstet Gynecol. 1999;94:66-70.

15. Ptaszkowski K, Paprocka-Borowicz M, Słupska L, et al. Assessment of bioelectrical activity of synergistic muscles during pelvic floor muscles activation in postmenopausal women with and without stress urinary incontinence: a preliminary observational study. Clin Interv Aging. 2015; 10:1521-1528.

16. Halski T, Słupska L, Dymarek R, et al. Evaluation of bioelectrical activity of pelvic floor muscles and synergistic muscles depending on orientation of pelvis in menopausal women with symptoms of stress urinary incontinence: a preliminary observational study. Biomed Res Int. 2014;2014: 274938.

17. Pietrangelo $T$, Mancinelli $R$, Toniolo $L$, et al. Effects of local vibrations on skeletal muscle trophism in elderly people: mechanical, cellular, and molecular events. Int J Mol Med. 2009;24:503-512.

18. lodice $P$, Bellomo RG, Gialluca G, et al. Acute and cumulative effects of focused high-frequency vibrations on the endocrine system and muscle strength. Eur J Appl Physiol. 2011;111:897-904.

19. Barber MD, Walters MD, Bump RC. Short forms of two condition-specific quality-of-life questionnaires for women with pelvic floor disorders (PFDI20 and PFIQ-7). Am J Obstet Gynecol. 2005;193:103-113.

20. Von Elm E, Altman DG, Egger $M$, et al. The Strengthening the Reporting of Observational Studies in Epidemiology (STROBE) statement: guidelines for reporting observational studies. Lancet. 2007;370: 1453-1457.

21. Van Deun B, Hobbelen JSM, Cagnie B, et al. Reproducible measurements of muscle characteristics using the MyotonPRO Device: comparison Between individuals with and without paratonia. J Geriatr Phys Ther. 2018;41:194-203.

22. Barassi G, Bellomo RG, Porreca A, et al. The use of adaptive neurostimulation for rebalancing posture and muscular tone in a soccer team. J Sports Med Phys Fitness. 2019 [Epub ahead of print]; DOI: 10.23736/ S0022-4707.19.09311-3.

23. Pruyn EC, Watsford ML, Murphy AJ. Validity and reliability of three methods of stiffness assessment. J Sport Health Sci. 2016;5: 476-483.

24. Barber MD, Chen $Z$, Lukacz E, et al. Further validation of the short form versions of the pelvic floor distress inventory (PFDI) and pelvic floor impact questionnaire (PFIQ). Neurourol Urodyn. 2011;30:541-546.

25. Saggini R, Ancona E, Carmignano, et al. Effect of combined treatment with focused mechano-acoustic vibration and pharmacological therapy on bone mineral density and muscle strength in post-menopausal women. Clin Cases Miner Bone Metab. 2017;14:305-311.

26. Aksac B, Aki S, Karan A, et al. Biofeedback and pelvic floor exercises for the rehabilitation of urinary stress incontinence. Gynecol Obstet Invest. 2003; $56: 23-27$.

27. Loewenstein WR, Skalak R. Mechanical transmission in a Pacinian corpuscle. An analysis and a theory. J Physiol. 1966;182:346-378.

28. Matthews PB, Watson JD. Action of vibration on the response of cat muscle spindle la afferents to low frequency sinusoidal stretching. J Physiol. 1981;317:365-381.

29. Fallon JB, Macefield VG. Vibration sensitivity of human muscle spindle and Golgi tendon organs. Muscle Nerve. 2007;36:21-29.

30. Marconi B, Filippi GM, Koch G, et al. Long-term effects on motor cortical excitability induced by repeated muscle vibration during contraction in healthy subjects. J Neurol Sci. 2008;275:51-59.

31. Liepert J, Classen J, Cohen LG, et al. Task-dependent changes of intracortical inhibition. Exp Brain Res. 1998;118:421-426.

32. Brunetti O, Filippi GM, Lorenzini M, et al. Improvement of posture stability by vibratory stimulation following anterior cruciate ligament reconstruction. Knee Surg Sports Traumatol Arthrosc. 2006;14: 1180-1187.

33. Silva MET, Brandão S, Parente MPL, et al. Biomechanical properties of the pelvic floor muscles of continent and incontinent women using an 
inverse finite element analysis. Comput Methods Biomech Biomed Eng. 2017;20:842-852.

34. Aljuraifani R, Stafford RE, Hall LM, et al. Task-specific differences in respiration-related activation of deep and superficial pelvic floor muscles. J Appl Physiol (1985). 2019;126:1343-1351.

35. Agyapong-Badu S, Warner M, Samuel D, et al. (2018). Practical Considerations for Standardized Recording of Muscle Mechanical Properties Using a Myometric Device: recording Site, Muscle Length, State of Contraction and Prior Activity. J Musculoskelet Res. 2018;21:1850010.

Cite this article as: Paolucci T, Bellomo RG, Pezzi L, Frondaroli F, Frondaroli S, Santarelli A, Barbato C, Porreca A, Saggini R (2019) A novel rehabilitative protocol in the treatment of mixed urinary incontinence in women: the effects of focused mechano-acoustic vibration, BioResearch Open Access 8:1, 219-228, DOI: 10.1089/ biores.2019.0041.

$\begin{aligned} & \quad \text { Abbreviations Used } \\ & \mathrm{AM}=\text { adductor magnus } \\ & \mathrm{BMI}=\text { body mass index } \\ & \mathrm{GM}=\text { gluteus maximus } \\ & \mathrm{GMD}=\text { gluteus maximus muscle logarithmic decrement } \\ & \mathrm{GMF}=\text { gluteus maximus muscle oscillation frequency } \\ & \mathrm{GMS}=\text { gluteus maximus muscle dynamic stiffness } \\ & \mathrm{MI}=\text { mixed incontinence } \\ & \mathrm{MUI}=\text { mixed urinary incontinence } \\ & \mathrm{PFDI}=\text { pelvic floor disability index } \\ & \mathrm{PFIQ}=\text { pelvic floor impact questionnaire } \\ & \mathrm{PFM}=\text { pelvic floor muscle } \\ & \mathrm{RA}=\text { rectus abdominis } \\ & \mathrm{SI}=\text { stress incontinence } \\ & \mathrm{SUI}=\text { stress urinary incontinence } \\ & \mathrm{UI}=\text { urge incontinence }\end{aligned}$

\section{Publish in BioResearch Open Access}

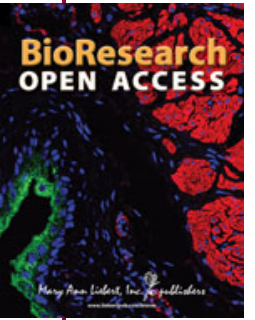

- Broad coverage of biomedical research - Immediate, unrestricted online access

- Rigorous peer review

- Compliance with open access mandates

- Authors retain copyright

- Highly indexed

- Targeted email marketing

liebertpub.com/biores 